\title{
A DEA-based Approach for Finding a Favorable Multi- Objective Shortest Path
}

\author{
Gholam Hassan Shirdel ${ }^{1, *}$ and Somayeh Ramezani-Tarkhorani ${ }^{1}$ \\ ${ }^{1}$ Faculty of Sciences, University of Qom, Alghadir Bld., Postal code: 3716146611, Qom, Iran \\ E-mail: <shirdel81math@gmail.com,sramezani_t@yahoo.com>
}

\begin{abstract}
Multi-objective shortest path problems deal with networks in which each are is associated with multiple types of costs and profits. The conditions can increase number of nondominated paths and compounds difficulty of selecting a favorable one for Decision Maker (DM). In this paper we suggest a hybrid method to generate a non-dominated path which fulfills decision maker's demands. It uses DEA in a more logical way than some previous DEA-based approaches. We take advantage of common weights methodology. DM can give relative importance of some cost and profit indicators. Eventually, we have a shortest path problem that can be solved with Dijkstra's algorithm. Two numerical examples are presented and the results are analyzed.
\end{abstract}

Keywords: common set of weights, data envelopment analysis, decision maker, multi objective shortest path problem, weight restrictions

Received: January 10, 2018; accepted: July 20, 2018; available online: December 13, 2018

DOI: 10.17535 /crorr.2018.0012

\section{Introduction}

The shortest path problem is one of the most important problems in network flows with wide range of applications in different areas (telecommunications, transportations, manufacturing, etc.). In these problems, each arc has just one cost. For example, if an arc represents a road, this cost can be the spent money or the amount of time needed for passing the road. The objective is to find a path with minimum cost or a path with fewest arcs; in traditional form of the shortest path problem, there is only one objective function. There are many algorithms to solve this problem including works of Dijkstra et al. [12] and Ahuja et al. [2]. But in real world, it is very likely that different types of costs be allocated to one arc. For example, in arc-road correspondence, one can divide the cost to spent money and time and add some profits like security, desirability, etc. Therefore, for moving from a node to another, we want to find a path which is the best solution respect to multiple objectives. That is why these problems are called

\footnotetext{
${ }^{*}$ Corresponding author.

http://www.hdoi.hr/crorr-journal

@2018 Croatian Operational Research Society
} 
multi-objective shortest path problems. In most cases, there is no optimal solution; therefore one seeks non-dominated (Pareto-optimal) solutions: solutions that cannot be replaced with the better ones. Martins et al. [20] presented two algorithms for MCSP problem. One of them was a generalization of multiple labeling schemes algorithm of Hansen [17] for the bi-criteria case, and the second one was the simplex method used in continuous linear multi-objective programming. There are some works as those have done by Guerriero et al. [15], Brumbaugh-Smith et al. [5], Mote et al. [23], Bertsekas et al. [4], Climaco et al. [8], Hamacher et al. [16], Current et al. [11], Modesti et al. [22], Coutinho-Rodrigues et al. [10], Murthy et al. [24], Duque et al. [13], SedenoNoda et al. [26], Sinha et al. [27]. DEA, firstly introduced by Charnes et al. [6], is a nonparametric method to assess relative performance of a group of homogeneous Decision Making Units (DMUs), it's explained more latter. Recently, some DEA-based methods have been suggested to generate non-dominated paths. Masoumi et al. [21] let each path from the source node to the sink one to be a DMU that had no output and with costs as input. They showed each non-dominated path is equivalent to a strongly efficient DMU and used FDH model. Amirteimoori [3] extended the classical shortest path problem by considering multiple attributes for each arc (as inputs and outputs). He obtained two measures for each arc by using DEA models and associated the average of two measures to the arc. Then, he defined the overall efficiency of each path, from the source node to the sink one. Finally, the efficient path, a path with maximum efficiency, was determined.

Moreover, some methods with the same approach have been proposed in assignment problem, we can mention some works as those have done by Zarafat Angiz et al. [29], Chen et al. [7], Emrouznejad et al. [14]. In this paper, we suggest a method where DM's points of view and DEA methodology will help us to find a non-dominated multiple objective shortest path. To accomplish that, first we convert DM's propensities to restrictions about weight of indicators and then we add them to a DEA model for generating a Common Set of Weights (CSW). Our purpose is to generate a CSW coming from involvement of DM's preferences and fairness in evaluation of all arcs. The latter is what puts distinction between our method and some other DEA-based methods where each arc or path is evaluated by its own weight. As matter of fact, it seems more reasonable that arcs and paths to be assessed under the same conditions. In our DEA-based method, we define a DMU for each arc. Since our overall intention is to decrease cost and increase profit, therefore cost and profit of an arc are assumed to be respectively input and output. Then, based on generated CSW, a virtual scalar cost is allocated to each arc. It transforms the problem to a simple shortest path problem. It turns out that the scalar criterion is non-negative and Dijkstra's algorithm or any shortest path algorithm can be used to solve it. This is essential from computational complexity angle. At the end, we prove that the path is non-dominated. In this paper, we briefly review the literature and required notions in section 2 and introduce the main problem in section 3. The proposed model, including its details, is discussed in section 4 . We, also, bring two numerical examples, discuss and compare their results in section 5 . 


\section{Background}

In this section, we mention some necessary definitions and theorems.

\subsection{Multi objective linear programming problem}

The general form of a Multi-objective Linear Programming problem can be represented as following:

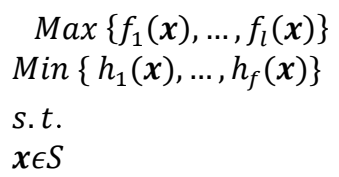

where $\mathrm{x}$ represents the vector of decision variables, $f_{r}(\boldsymbol{x})(r=1, \ldots, l)$ and $h_{i}(\boldsymbol{x})(i=1, \ldots, f)$ are linear objective functions, $S=\left\{\boldsymbol{x} \mid g_{j}(\boldsymbol{x}) \leq b_{j}, j=1, \ldots, k\right\}$ and $g_{j}(\boldsymbol{x})(j=1, \ldots, k)$ are linear functions and $b_{j} \in \mathbb{R}(j=1, \ldots, k)$.

A non-dominated solution (Pareto-optimal solution) for the above problem defines as following:

Definition1: $\bar{x} \epsilon S$ is a non-dominated (Pareto-optimal) solution for (1) if it satisfies following conditions:

$$
\exists ! \boldsymbol{x} \in S:\left(f_{r}(\boldsymbol{x}) \geq f_{r}(\overline{\boldsymbol{x}})(r=1, \ldots, l), h_{i}(\boldsymbol{x}) \leq h_{i}(\overline{\boldsymbol{x}})(i=1, \ldots, f),\right.
$$

where there is at least one strict inequality.

The following Theorem states one of methods for finding a non-dominated solution to (1):

Theorem 1. Let $\alpha_{r} \in \mathbb{R}^{+}(r=1, \ldots, l)$ and $\beta_{i} \in \mathbb{R}^{+}(i=1, \ldots, m)$. Then, the optimal solution of (3) is a non-dominated solution for (1).

$$
\begin{aligned}
& \operatorname{Min} y(\boldsymbol{x})=\sum_{i=1}^{f} \beta_{i} h_{i}(\boldsymbol{x})-\sum_{r=1}^{l} \alpha_{r} f_{r}(\boldsymbol{x}) \\
& \quad \text { s.t. } \\
& \quad \boldsymbol{x} \in S
\end{aligned}
$$

Proof. [18]

\subsection{Data Envelopment Analysis}

DEA is a quantitative non-parametric method that uses mathematical programming for measuring and evaluating relative performance of a group of homogeneous Decision Making 
Units with the same types of inputs and outputs. Therefore, it has grown into a powerful management tool in operations research and economics.

Let $x_{i j}$ denotes the data of $i^{t h}(i=1, \ldots, f)$ input for $D M U_{j}$ and $y_{i j}$ denotes the data of $r^{\text {th }}(r=1, \ldots, l)$ output for it.

Let $(\boldsymbol{u}, \boldsymbol{v})$ be an arbitrary set of weights where $\mathbf{u}^{\mathrm{t}} \in \mathbb{R}^{\mathrm{l}}, \mathbf{v}^{\mathrm{t}} \in \mathbb{R}^{\mathrm{f}}$.

The absolute efficiency of $D M U_{o}(o \in\{1, \ldots, n\})$, based on $(\boldsymbol{u}, \boldsymbol{v})$, is:

$$
\frac{\sum_{r=1}^{l} u_{r} y_{r o}}{\sum_{i=1}^{f} v_{i} x_{i o}}
$$

The (5) shows multiplier form of input oriented CCR model for evaluating $D M U_{o}$ is as follows:

$$
\begin{gathered}
\theta_{0}^{*}=\operatorname{Max} \boldsymbol{u} \boldsymbol{y}_{\boldsymbol{o}} \\
\text { s.t. } \\
\boldsymbol{v} \boldsymbol{x}_{\boldsymbol{o}}=1 \\
\boldsymbol{u} \boldsymbol{y}_{\boldsymbol{j}}-\boldsymbol{v} \boldsymbol{x}_{\boldsymbol{j}} \leq \mathbf{0} \quad j=1, \ldots, n \\
\boldsymbol{u} \geq \mathbf{0}, \boldsymbol{v} \geq \boldsymbol{o}
\end{gathered}
$$

where, the decision variables $\boldsymbol{v} \in \mathbb{R}^{f}$ and $\boldsymbol{u} \in \mathbb{R}^{l}$ represent respectively the input weights and output weights. $\boldsymbol{x}_{\boldsymbol{j}} \in \mathbb{R}^{f}$ and $\boldsymbol{y}_{\boldsymbol{j}} \in \mathbb{R}^{l}(j=1, \ldots, n)$ indicate respectively the vector of inputs and the vector of outputs for $D M U_{j}$.

Dual of (5) is (6) that is called envelopment form of input oriented CCR model for assessment of $D M U_{0}$.

$$
\begin{aligned}
& \theta_{0}^{*}=\min \theta \\
& \quad \text { s.t. } \\
& \sum_{j=1}^{n} \lambda_{j} \boldsymbol{x}_{j} \leq \theta \boldsymbol{x}_{\boldsymbol{o}} \\
& \sum_{j=1}^{n} \lambda_{j} \boldsymbol{y}_{j} \geq \boldsymbol{y}_{\boldsymbol{o}} \\
& \lambda_{j} \geq 0 \quad j=1, \ldots, n \\
& \quad \theta \text { is free }
\end{aligned}
$$

where, the $\lambda_{j}(j=1, \ldots, n)$ and $\theta$ are decision variables. $\boldsymbol{x}_{j} \in \mathbb{R}^{f}$ and $\boldsymbol{y}_{j} \in \mathbb{R}^{l}(j=1, \ldots, n)$ indicate respectively the vector of inputs and the vector of outputs for $D M U_{j}$. It turns out that $\theta_{0}^{*} \leq 1$. If $\theta_{0}^{*}=1$, then $D M U_{0}$ is efficient and otherwise inefficient.

In real problems, extra information may be lead to imposing some weight restrictions on DEA models. Assurance region (AR) method, developed by Thompson et al. [28], impose the weight restrictions stating the lower and upper bounds for ratio of some input and output weights.

Numerous types of ranking methods have been suggested by researchers for distinctions among performance of efficient DMUs. Common weights methodology was introduced by Cook et al. [9] and Roll et al. [25]. In general, ranking methods with common set of weights can provide evaluation of units under equal conditions. Therefore, it is fairer. It is also worth to mention that most of these methods are able to rank all the DMUs (e.g. [12]). 


\section{Problem definition}

Suppose $G=(V, A)$ be a directed graph where $V$ and $A$, respectively, indicate the set of nodes and the set of $\operatorname{arcs}$ in $\mathrm{G},|\mathrm{A}|=\mathrm{m}$. $(i, j) \in A$ if and only if there is an arc from ith node to jth node in the network. Let $N=(G, \boldsymbol{c}, \boldsymbol{p}, s, t)$ be a network with distinguished nodes $\mathrm{s}$ and $\mathrm{t}$ as respectively the source node and destination (sink) node. $\mathrm{c}$ and p represent the arc weights. In fact, there are a finite number of indicators to evaluate each arc. for conveniences, we call those DM wishes to be increased, profit indicators and those he/she wishes to be decreased, cost indicators.

It's assumed that the indicators satisfy the following assumptions:

- Number and types of allocated indicators are equal for all the arcs.

- There are $\mathrm{f}$ cost indicators and 1 profit indicators.

- When a unit of flow passes through $\operatorname{arc}(i, j) \epsilon A$, amount of the $k^{\text {th }}(k=1, \ldots, f)$ type of $\operatorname{cost}$ is $c_{i j}^{k}$ and it is a non-negative integer number.

- When a unit of flow passes through arc $(i, j) \epsilon A$, amount of the $r^{\text {th }}(r=1, \ldots, l)$ type of profit is $p_{i j}^{r}$ and it is a non-negative integer number.

- $\quad \forall(i, j) \in A\left(\exists k \epsilon\{1, \ldots f\}: c_{i j}^{k}>0\right)$ and $\left(\exists r \epsilon\{1, \ldots l\}: p_{i j}^{r}>0\right)$.

Let $\mathcal{P}_{s t}$ denotes the set of all path from s to t (s-t paths) in graph $\mathrm{G}$ and $P \in \mathcal{P}_{s t}$. When a unit of flow passes through $P$, amounts of costs and profits are respectively, $c_{k}(P)=$ $\sum_{(i, j) \in A(P)} c_{i j}^{k}(k=1, \ldots, f)$ and $\mathrm{p}_{r}(P)=\sum_{(i, j) \epsilon A(P)} p_{i j}^{r}(r=1, \ldots l)$ where $\mathrm{A}(\mathrm{P})$ is the set of arcs in path P.

Definition 2. $\bar{P} \in \mathcal{P}_{\text {st }}$ is a non-dominated (Pareto-optimal) s-t path in graph $G$ if satisfies following conditions:

$$
\exists ! P \in \mathcal{P}_{s t}:\left(p_{r}(P) \geq p_{r}(\bar{P})(r=1, \ldots, l), c_{i}(P) \leq c_{i}(\bar{P})(i=1, \ldots, f),\right.
$$

where there is at least one strict inequality.

Our objective is to find a s-t path so that when a unit of flow passes through it, amount of each indicator be optimum comparing to other s-t paths. It means amount of cost indicators are minimum and the amount of profit indicators are maximum. We call this s-t path an optimal s-t path.

Now we are ready to formulate this Multi Objective Shortest Path Problem as follows:

$$
\begin{aligned}
& \max \left\{\sum_{(\mathrm{i}, j) \in \mathrm{A}} \mathrm{p}_{\mathrm{ij}}^{1} \mathrm{x}_{\mathrm{ij}}, \ldots, \sum_{(\mathrm{i}, \mathrm{j}) \in \mathrm{A}} \mathrm{p}_{\mathrm{ij}}^{1} \mathrm{x}_{\mathrm{ij}}\right\} \\
& \min \left\{\sum_{(\mathrm{i}, \mathrm{j}) \in \mathrm{A}} \mathrm{c}_{\mathrm{ij}}^{1} \mathrm{x}_{\mathrm{ij}}, \ldots, \sum_{(\mathrm{i}, \mathrm{j}) \in \mathrm{A}} \mathrm{c}_{\mathrm{ij}}^{\mathrm{f}} \mathrm{x}_{\mathrm{ij}}\right\} \\
& \text { s.t. } \\
& \sum_{\mathrm{j} \in \mathrm{N}^{+}(i)} \mathrm{x}_{\mathrm{ij}}-\sum_{j \in N^{-}(i)} x_{\mathrm{ji}}=\left\{\begin{array}{cc}
1 & \mathrm{i}=\mathrm{s} \\
0 & \mathrm{i} \neq \mathrm{s}, \mathrm{t} \\
-1 & \mathrm{i}=\mathrm{t}
\end{array}\right. \\
& \mathrm{x}_{\mathrm{ij}} \in\{0,1\} \quad(i, j) \in A
\end{aligned}
$$


where, for each $i \epsilon V, \quad N^{+}(i)=\{j \mid(i, j) \epsilon \mathrm{A}\}$ and $N^{-}(i)=\{j \mid(j, i) \epsilon \mathrm{A}\}$. All $\mathrm{x}_{\mathrm{ij}} \in A$ are decision variables. Foe each $(i, j) \epsilon A, p_{i j}^{r}(r=1, \ldots, l)$ and $\mathrm{c}_{\mathrm{ij}}^{\mathrm{k}}(\mathrm{k}=1, \ldots, \mathrm{f})$ are respectively the profit and cost parameters.

\section{A Method for Determining a Favorable Non-dominated s-t Path}

Here, we propose a DEA-based approach to introduce a favorable non-dominated s-t path to DM. In comparison with some former works in this area, we attain to utilizing DEA approach in a more logical way. To observe equal and fair conditions, we use common weights methodology to evaluate the ares of network and consequently the s-t paths. It is also worth in terms of computational aspect. In the following, we elaborate the process.

\subsection{Step one: Network as a DEA structure}

Consider $N=(G, c, p, s, t)$ with the prior mentioned characteristics (Section 3). Firstly, we correspond each arc $(i, j) \in A$ to a decision making unit $\mathrm{DMU}_{i j}$ for which the consumed inputs and produced outputs vectors are equal to the cost and profit vectors of the arc, respectively. That means $\left[\mathrm{x}_{\mathrm{ij}}^{1}, \ldots, \mathrm{x}_{\mathrm{ij}}^{f}\right]=\left[\mathrm{c}_{\mathrm{ij}}^{1}, \ldots, \mathrm{c}_{\mathrm{ij}}^{\mathrm{f}}\right]$ and $\left[\mathrm{y}_{\mathrm{ij}}^{1}, \ldots, \mathrm{y}_{\mathrm{ij}}^{l}\right]=\left[\mathrm{p}_{\mathrm{ij}}^{1}, \ldots, \mathrm{p}_{\mathrm{ij}}^{1}\right]$, where $\mathrm{x}_{\mathrm{ij}}^{k}(k=1, \ldots, f)$ and $y_{\mathrm{ij}}^{r}(r=$ $1, \ldots, s)$ denotes respectively amounts of the $i^{\text {th }}$ input and the $r^{\text {th }}$ output for $\mathrm{DMU}_{i j}$. Given a set of weighs $(u, v)$, where $u^{t} \in \mathbb{R}_{\geq 0}^{l}$ and $v^{t} \in \mathbb{R}_{\geq 0}^{f}$, the weighted sum of the inputs (costs), $\sum_{k=1}^{\mathrm{f}} v_{k} c_{i j}^{k}$, is considered as a virtual input (cost) and the weighted sum of outputs (profits), $\sum_{\mathrm{r}=1}^{\mathrm{l}} u_{\mathrm{r}} \mathrm{p}_{\mathrm{ij}}^{r}$, is considered as a virtual output (profit).

\subsection{Step Two: Generating a Common Set of Weights}

Here, we discuss how a DEA model will be part of finding a favorable s-t path. Definition of the scalar criterion to evaluate arcs (to be discussed later) indicates that the weights used play an essential role in its computation. Hence, we take advantage of common weights methodology in DEA. Also, we want to involve the DM's points of view in process of choosing the CSW and eventually the obtained non-dominated s-t path. Having said that, we explain how to obtain a common set of weights in two steps:

i. Dealing with DM's Preferences

ii. Using a DEA model to generate a CSW.

\subsubsection{Dealing with DM's Preferences}

In our method, DM is asked to determine relative importance of the cost and profit factors, if there are any. $\mathrm{He} /$ she is not forced to give an exact value; giving bounds of it (at least one) will work in this case. DM's points of view will appear in the model as weight restrictions. Let $i \neq j$ be two arbitrary indicators that DM intends to express his preferences through them. The following formula is generally how we consider the DM's intuition: 


$$
\xi_{i j} \leq \frac{\text { weight of } \text { ith indicator }}{\text { weight of } \text { jth indicator }} \leq \bar{\zeta}_{i j}
$$

where satisfies the following:

- It is possible that the indicators be of any type (cost or profit).

- $\xi_{i j} \in \mathbb{R}^{+} \cup\{0\}, \bar{\xi}_{i j} \in \mathbb{R}^{+} \cup\{+\infty\}$

- If DM only determines the lower bound $\xi_{i j}$, then $\bar{\xi}_{i j}$ is set to $+\infty$.

- If DM only determines the upper bound $\bar{\xi}_{i j}$, then $\xi_{i j}$ is set to be 0 .

- $\xi_{i j} \leq \bar{\xi}_{i j}$, the equation occurs when DM state the relative importance exactly.

It is worth to mention that DM is supposed to logically assign bounds. It means that, for example, if indicator $i$ is more important than indicator $j$ (for any type of cost or profit) then $\xi_{i j}$ must be greater than 1. Also, at most one formula for each pair of factors is considered. In Section 5, we discuss several different cases and investigate alteration of the parameters and its effects on the final results.

\subsubsection{Using a DEA model to generate a CSW}

Here, we present a DEA model using assurance region method to generate a CSW. This model has been influenced by Liu and Peng's work [19] which is computationally simple to use. The aim behind the model is to maximize the efficiencies of DMUs by maximizing their summation. The assurance region restrictions carry DM's points of view. The main model to generate a CSW, in a general form, comes as follows:

$$
\begin{aligned}
& \operatorname{Max} \sum_{(i, j) \in A} \frac{\sum_{\mathrm{r}=1}^{\mathrm{l}} \mathrm{u}_{\mathrm{r}} \mathrm{p}_{\mathrm{ij}}^{\mathrm{r}}}{\sum_{\mathrm{k}=1}^{\mathrm{f}} \mathrm{v}_{\mathrm{k}} \mathrm{c}_{\mathrm{ij}}^{\mathrm{k}}} \\
& \text { s.t. } \\
& \frac{\sum_{\mathrm{r}=1}^{\mathrm{l}} \mathrm{u}_{\mathrm{r}} \mathrm{p}_{\mathrm{ij}}^{\mathrm{r}}}{\sum_{\mathrm{k}=1}^{f} \mathrm{v}_{\mathrm{k}} \mathrm{c}_{i j}^{\mathrm{k}}} \leq 1 \quad \forall(\mathrm{i}, \mathrm{j}) \in \mathrm{A} \\
& \alpha_{\mathrm{kk}^{\prime}} \leq \frac{\mathrm{v}_{\mathrm{k}}}{\mathrm{v}_{\mathrm{k}^{\prime}}} \leq \bar{\alpha}_{\mathrm{kk}^{\prime}} \quad \forall\left(\mathrm{k}, \mathrm{k}^{\prime}\right) \in \Gamma_{I} \\
& \beta_{\mathrm{rr}} \leq \frac{\mathrm{u}_{\mathrm{r}}}{\mathrm{u}_{\mathrm{r}^{\prime}}} \leq \bar{\beta}_{\mathrm{rr}^{\prime}} \quad \forall\left(\mathrm{r}, \mathrm{r}^{\prime}\right) \in \Gamma_{O} \\
& \mathrm{v}_{\mathrm{rk}} \leq \frac{\mathrm{v}_{\mathrm{i}}}{\mathrm{u}_{\mathrm{r}^{\prime}}} \leq \overline{\mathrm{r}}_{\mathrm{rk}} \quad \forall(\mathrm{r}, \mathrm{k}) \in \Gamma_{I O} \\
& \begin{array}{lr}
\quad \Delta_{\mathrm{ij}} \geq 0 & \forall(\mathrm{i}, \mathrm{j}) \in \mathrm{A} \\
\mathrm{u}_{\mathrm{r}} \geq \varepsilon & \mathrm{r}=1, \ldots, 1 \\
\mathrm{v}_{\mathrm{k}} \geq \varepsilon & \mathrm{k}=1, \ldots, \mathrm{f}
\end{array}
\end{aligned}
$$

(11) is solved instead of (10) (Refer to [13] for more explanations).

$$
\begin{aligned}
& \operatorname{Min} \sum_{(i, j) \in A} \Delta_{\mathrm{ij}} \\
& \text { s.t. } \\
& \sum_{\mathrm{r}=1}^{\mathrm{s}} \mathrm{u}_{\mathrm{r}} \mathrm{p}_{\mathrm{ij}}^{\mathrm{r}}-\sum_{\mathrm{k}=1}^{\mathrm{f}} \mathrm{v}_{\mathrm{k}} \mathrm{c}_{\mathrm{ij}}^{\mathrm{k}}+\Delta_{\mathrm{ij}}=0 \quad \forall(\mathrm{i}, \mathrm{j}) \in \mathrm{A} \\
& \left.\begin{array}{l}
-v_{k}+\alpha_{\mathrm{kk}^{\prime}} \mathrm{v}_{\mathrm{k}^{\prime}} \leq 0 \\
v_{k}-\bar{\alpha}_{\mathrm{kk}^{\prime}} \mathrm{v}_{\mathrm{k}^{\prime}} \leq 0
\end{array}\right\} \quad \forall\left(\mathrm{k}, \mathrm{k}^{\prime}\right) \in \Gamma_{I} \\
& -\mathrm{u}_{\mathrm{r}}+\beta_{\mathrm{rr}^{\prime}} \mathrm{u}_{\mathrm{r}^{\prime}} \leq 0 \\
& \mathrm{u}_{\mathrm{r}}-\bar{\beta}_{\mathrm{rr}} \mathrm{u}_{\mathrm{r}^{\prime}} \leq 0 \\
& \forall\left(r, r^{\prime}\right) \in \Gamma_{O}
\end{aligned}
$$




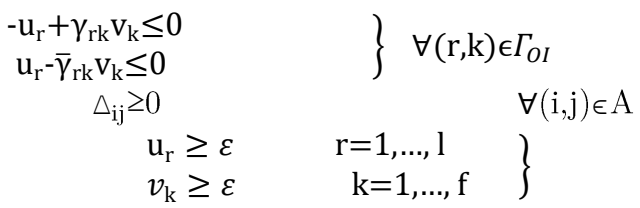

where, $v_{k}(k=1, \ldots, f), u_{r}(r=1, \ldots, l)$ and $\Delta_{i j}(i, j) \epsilon A$ are decision variables. For each $(i, j) \epsilon A, \mathrm{c}_{\mathrm{ij}}^{\mathrm{k}}(k=1, \ldots, f)$ and $\mathrm{p}_{\mathrm{ij}}^{\mathrm{r}}(r=1, \ldots, l)$ are respectively cost and profit parameters. The constraints of (1) guarantee that the efficiency of each DMU don't exceed one. The constraints of (2), (3) and (4) state weight restrictions about the relative importance and priority of cost and profit indicators. $\Gamma_{\mathrm{I}}$ denotes the set of the ordered pairs of input indicators' indexes which DM has given some information about their relative importance. This set satisfy the following properties: if $(i, k) \epsilon \Gamma_{\mathrm{I}}$ then $(k, i) \notin \Gamma_{\mathrm{I}}$; for all $i \epsilon V,(i, i) \notin \Gamma_{\mathrm{I}}$. Plus, there are similar statements about $\Gamma_{\mathrm{O}}$ (related to output indicators' indexes) and $\Gamma_{\mathrm{IO}}$ (related to input and output indicators' indexes). The weight restrictions are given in a general form regarding to (9). The parameters $\alpha_{\mathrm{kk}^{\prime}}$ and $\bar{\alpha}_{\mathrm{kk}}\left(\forall\left(\mathrm{k}, \mathrm{k}^{\prime}\right) \in \Gamma_{\mathrm{I}}\right)$ are respectively the lower and upper bounds of the relative importance of the input indicator $\mathrm{k}$ to $\mathrm{k}^{\prime}$ and the parameters $\beta_{r r}$, and $\bar{\beta}_{r r}\left(\forall\left(\mathrm{r}, \mathrm{r}^{\prime}\right) \in \Gamma_{\mathrm{O}}\right)$ are respectively the lower and upper bounds of the relative importance of the output indicator $\mathrm{r}$ to $\mathrm{r}^{\prime}$. Also, $\gamma_{\mathrm{rk}}$ and $\bar{\gamma}_{\mathrm{rk}}\left(\forall(\mathrm{r}, \mathrm{k}) \in \Gamma_{\mathrm{IO}}\right)$ are respectively the lower and upper bounds of the relative importance of the output indicator $r$ to input indicator $k$. However, practically, the weight restrictions of (2), (3) and (4) stands only for positive finite bounds (the rest are redundant, and so they are removed). All of these parameters are determined by DM. If he/she has no preference then we do not use the weight restrictions. $\varepsilon$ is a positive Archimedean infinitesimal constant. The batch constraints (6) guaranties that all weights in feasible solutions are non-zero.

Assume that $\left(\mathrm{u}^{*}, \mathrm{v}^{*}, \Delta^{*}\right)$ be the optimal solution of $(11)$. We accept $\left(\mathrm{u}^{*}, \mathrm{v}^{*}\right)$ as the common set of weights. Also, (11) can be rewritten as (12):

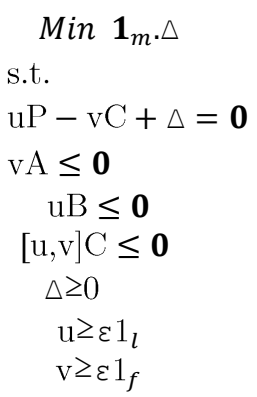

where, $\Delta \in \mathbb{R}^{m}, \boldsymbol{u}^{t} \in \mathbb{R}^{l}$ (output variables), $\boldsymbol{v}^{t} \in \mathbb{R}^{f}$ (input variables) are vectors of decision variables. $\mathrm{C}=\left[c_{\mathrm{ij}}\right]_{\mathrm{f} \times \mathrm{m}}$ and $\mathrm{P}=\left[p_{\mathrm{ij}}\right]_{\mathrm{l} \times \mathrm{m}}$ denote respectively the matrixes of arc costs and arc profits. $\mathrm{A} \in \mathbb{R}_{f \times a}, \mathrm{~B} \in \mathbb{R}_{l \times b}$ and $\mathrm{C} \in \mathbb{R}_{(l+f) \times h}$ contains the multipliers of assurance region constrains of (11). Regarding to the previous explanations about the bounds of weight ratios derived from DM's preferences, it is evident that: $\left|\Gamma_{I}\right| \leq a \leq 2\left|\Gamma_{I}\right|,\left|\Gamma_{O}\right| \leq b \leq 2\left|\Gamma_{O}\right|$ and $\left|\Gamma_{O I}\right| \leq h \leq 2\left|\Gamma_{O I}\right|$. For above inequalities, the minimum occur when there is no weight ratio with two positive finite bounds, 
and the maximum occur when both of lower and upper bounds of for the weight ratios are positive and finite.

In short, the interaction with DM is placed in this part and it finally leads to choosing a favorable s-t path based on DM preferences.

\subsection{Determining the Scalar Criterion}

Now, we use a scalar criterion to assess each arc. Let $\left(\mathrm{u}^{*}, \mathrm{v}^{*}\right)$ be the generated CSW. We consider the following scalar criterion, obtained based on the CSW, for each $\operatorname{arc}(\mathrm{i}, \mathrm{j}) \in \mathrm{A}$ :

$$
\lambda_{\mathrm{ij}}=\sum_{\mathrm{k}=1}^{\mathrm{f}} \mathrm{v}_{\mathrm{k}}^{*} \mathrm{c}_{\mathrm{ij}}^{\mathrm{k}}-\sum_{\mathrm{r}=1}^{\mathrm{l}} \mathrm{u}_{\mathrm{r}}^{*} \mathrm{p}_{\mathrm{ij}}^{\mathrm{r}}
$$

When a unit of flow passes through an arc $(\mathrm{i}, \mathrm{j})$, the more amounts of profits and the less amounts of costs make that arc more desirable for DM. Therefore according definition of (13), a lesser $\lambda_{\mathrm{ij}}$ is more favorable; it puts the scalar in the cost category. We allocate these scalars to arcs of the network as virtual costs. The constrains (1) of model (11) guarantee that $\lambda_{\mathrm{ij}} \geq 0(\forall(\mathrm{i}, \mathrm{j}) \in \mathrm{A})$. Therefore, each arc is allocated a non-negative virtual cost instead of several costs and profits. Now, let $P \in \mathcal{P}_{s t}$ (the set of all s-t paths) be an arbitrary s-t path. We define

$$
\lambda(\mathrm{P})=\sum_{(\mathrm{i}, \mathrm{j}) \in A(\mathrm{P})} \lambda(\mathrm{i}, \mathrm{j})
$$

where $\mathrm{A}(\mathrm{P})$ is the set of all arcs of $\mathrm{P}$. According to above discussion, a lesser $\lambda(\mathrm{P})$ is more favorable for DM, therefore, we intend to find a s-t path $\mathrm{P}$ with minimum amount of $\lambda(\mathrm{P})$.

\subsection{Finding a Favorable s-t Path}

In (4.3), each s-t path $\mathrm{P}$ is associated with a virtual scalar cost $\lambda(\mathrm{P})$. In this section, we aim to find a shortest s-t path respect to the virtual cost as a favorable s-t path. Thus, the following shortest path problem is solved:

$$
\begin{aligned}
& \min \sum_{(i, j) \epsilon A} \lambda_{i j} x_{i j} \\
& \sum_{x_{i j \in \mathrm{N}^{+}(\mathrm{i})} \in\{0,1\}} \mathrm{x}_{\mathrm{ij}}-\sum_{\left(\mathrm{j} \in \mathrm{N}^{-}(\mathrm{i})\right.} \mathrm{x}_{\mathrm{ji}}= \begin{cases}1 & \mathrm{i}=\mathrm{s} \\
0 & \mathrm{i} \neq \mathrm{s}, \mathrm{t} \\
-1 & \mathrm{i}=\mathrm{t}\end{cases}
\end{aligned}
$$

where $x_{i j}(i, j) \epsilon A$ are the decision variables and $\lambda_{i j}(i, j) \epsilon A$ are the cost parameters. It is evident that each shortest path from the source node $s$ to the destination node $t$ corresponds to an optimal solution to (15) [1]. 
As mentioned in (4.3), the virtual scalar cost allocated to each arc is non-negative so one can use Dijkstra's algorithm for obtaining a shortest s-t path. Theorem 3 guaranties that the obtained s-t path is non-dominated which is a worthwhile point.

Theorem 3. A s-t path $P$ with minimum $\lambda(P)$ is non-dominated.

Proof: The batch constraints (6) of (11) guarantees that the components of the generated CSW are positive, so the remained proof is easy.

\section{Numerical examples}

In this section, we apply our method on two numerical examples and investigate the results. The first example is relatively small because small-sized problems allow the details to be thoroughly discussed.

Example 1. Consider $N=(G, \boldsymbol{c}, \boldsymbol{p}, s, t)$ illustrated in Figure $1 . \mathrm{G}=(\mathrm{V}, \mathrm{A})$ is a directed graph where $V$ is the set of nodes and $A$ is the set of $\operatorname{arcs}(V=\{1,2, \ldots, 13\}$ and $|A|=19)$. In the network for passing of each unit of flow from each arc $(i, j)$, there are two types of expense, $c_{i j}^{1}$ and $c_{\mathrm{ij}}^{2}$, and one type of profit, $\mathrm{p}_{\mathrm{ij}}^{1}$. The source node, $\mathrm{s}$, is set to node labeled by 1 and the destination node, $\mathrm{t}$, is set to node labeled by 13 .

Table 1 demonstrates all the s-t paths of the network, values of costs, values of profits and state of each path including weather it is dominated and paths dominate it. We denote set of dominant paths on path $\mathrm{P}$ with $\mathrm{D}(\mathrm{P})$. We put some weight restrictions for the example and afterwards generate the CSW in each case (Table 2). In each case, using generated CSW, $\lambda_{\mathrm{ij}}$ is computed for each arc $(i, j)$ and then value of $\lambda(P)$ is determined for each s-t path p (Table 3 ). Table 4 shows the shortest s-t paths in each case. Also in order to do better comparison, we sort s-t paths in each case by value of $\lambda(\mathrm{P})$. The path with the least $\lambda(\mathrm{P})$ outranks others (Table 5).

In this example, coefficients are more diverse and their influence on the generated CSWs is recognizable. In each case, indicators which weigh more play a greater role in computation of $\lambda$. For example, in case 1 , since weight of cost in the second type is more than it in the first type, one unit difference in the second type is more effectively than one unit difference in the first type. It is why $\lambda(\mathrm{P} 1)$ is less than $\lambda(\mathrm{P} 3)$ and consequently P1 outranks P3. Theoretically, $\lambda(\mathrm{P})$ for a dominated s-t path $\mathrm{P}$ is more than a s-t path which dominates it and therefore ranks lower. Table 5 indicates that in this method a dominated s-t path can outranks some non-dominated s-t paths, for instance P2. It does not contradict the results because mentioned relation is between a dominated s-t path and the s-t paths dominated it. For example, P2 is dominated by P1. However, this path, in some indicators, is better than some of non-dominated paths like P4 and P7. It concludes, when all the cost and profit indicators are evaluated under a CSW, a dominated s-t path can have better rank than a non-dominated s-t path which does not dominate it. Finally, for a better illustration, Figure 2 shows $\lambda(\mathrm{P})$ for each 
s-t path, P, in each case, and Figure 3 shows the ranking scores of all s-t paths with all cases of weight restrictions.

Example 2. This example is bigger and has thirty nodes and one hundred arcs. Three cost and three profit indicators are considered to evaluate the arcs. This network can be assumed as a road network where nodes are cities and each arc $(i, j)$ is a route between city i and city $j$. In this way, it can be assumed that $1^{\text {th }}, 2^{\text {th }}$ and $3^{\text {th }}$ cost indicators are respectively fuel consumption, travel time and expenses that $1^{\text {th }}, 2^{\text {th }}$ and $3^{\text {th }}$ profit indicators are respectively earned income, safety factor and desirability. Therefore, the purpose is finding a favorable path between city 1 (the source node) and city 23 (the destination node). Since different people may have different priorities when it comes to road trips, various cases are considered for level of importance of indicators. In each case, we determine weight restrictions and compute the correspondent CSW (Table 6). Table 7 present an obtained favorable s-t path for each case, $P^{*(i)}$ for case i. For comparison, Table 8, considering all the cases, demonstrates the virtual costs for each of the favorable s-t paths. It is worth to point out that although in each case role of indicators with more relative importance is undeniable in selecting the favorable s-t path, but one cannot ignore the other indicators. There are cases where values of these indicators nullify effects of indicators with high relative importance. Comparison of and $P^{*^{(2)}}$ accentuate this matter. Interestingly, in case 2 importance, and therefore influence, of the first type profit is more third type cost. Here, regarding first type profit, $P^{*^{(1)}}$ is better than $P^{*^{(2)}}$ but not in the third type cost (Table 7 ). Although, preference of the first type profit to the third type cost could not prevent $P^{*^{(2)}}$ from outranking $P^{*(1)}$. 


\begin{tabular}{|l|l|l|l|l|l|}
\hline & \multicolumn{3}{|c|}{ Costs and profit } & \multicolumn{2}{c|}{ Non-dominancy status } \\
\hline P & $\mathrm{c} 1(\mathrm{P})$ & $\mathrm{c} 2(\mathrm{P})$ & $\mathrm{p} 1(\mathrm{P})$ & non-dominated & \multicolumn{1}{c|}{$\mathrm{D}(\mathrm{P})$} \\
\hline P1 & 12 & 11 & 10 & $\sqrt{ }$ & - \\
\hline P2 & 12 & 13 & 10 & $\times$ & - \\
\hline P3 & 11 & 12 & 10 & $\sqrt{ }$ & - \\
\hline P4 & 10 & 14 & 6 & $\sqrt{ }$ & - \\
\hline P5 & 15 & 13 & 9 & $\sqrt{ }$ & P1,P2,P3,P5 \\
\hline P6 & 16 & 15 & 9 & $\times$ & - \\
\hline P7 & 9 & 20 & 10 & $\sqrt{ }$ & \\
\hline
\end{tabular}

Table 1: $s$-t paths $(s=1, t=13)$ and their characteristics

\begin{tabular}{|l|l|c|}
\hline Case 1 & $\begin{array}{c}\text { Weight restrictions } \\
\mathrm{v} 2=2 \mathrm{v} 1, \quad \mathrm{u} 1=\mathrm{v} 1\end{array}$ & $(0.000100,0.000200,0.000100)$ \\
\hline Case 2 & $\mathrm{v} 2=2 \mathrm{v} 1$ & $(0.000100,0.000200,0.000167)$ \\
\hline Case 3 & $\mathrm{v} 2=5 \mathrm{v} 1$ & $(0.000100,0.000500,0.000367)$ \\
\hline Case 4 & $\mathrm{v} 2 \geq \mathrm{v} 1$ & $(0.000100,0.000100,0.000100)$ \\
\hline Case 5 & $\mathrm{v} 2 \geq 2 \mathrm{v} 1 \quad(0.000100,0.000200,0.000167)$ \\
\hline Case 6 & $\mathrm{v} 2 \geq 2 \mathrm{v} 1 \quad, 3 \mathrm{v} 1 \leq \mathrm{u} 1 \leq 5 \mathrm{v} 2$ & $(0.000100,0.000400,0.000300)$ \\
\hline
\end{tabular}

Table 2: The weight restrictions and generated CSWs

\begin{tabular}{|l|c|c|c|c|c|c|}
\hline s-t path & Case1 & Case2 & \multicolumn{1}{c|}{ Case3 } & \multicolumn{1}{c|}{ Case4 } & \multicolumn{1}{c|}{ Case5 } & \multicolumn{1}{c|}{ Case6 } \\
\hline P1 & 0.0024 & 0.00173 & 0.00303 & 0.0013 & 0.00307 & 0.0026 \\
\hline P2 & 0.0028 & 0.00213 & 0.00403 & 0.0015 & 0.00347 & 0.0034 \\
\hline P3 & 0.0025 & 0.00183 & 0.00343 & 0.0013 & 0.00317 & 0.0029 \\
\hline P4 & 0.0032 & 0.02798 & 0.005798 & 0.0018 & 0.003602 & 0.0048 \\
\hline P5 & 0.0032 & 0.02597 & 0.004697 & 0.0019 & 0.003803 & 0.004 \\
\hline P6 & 0.0037 & 0.03097 & 0.005797 & 0.0022 & 0.004303 & 0.0049 \\
\hline P7 & 0.0039 & 0.00323 & 0.00723 & 0.0019 & 0.00357 & 0.0059 \\
\hline
\end{tabular}

Table 3: $\lambda(P)$ for $s$-t paths

\begin{tabular}{|l|l|}
\hline & P* \\
\hline Case1 & P1 \\
\hline Case2 & P1 \\
\hline Case3 & P1 \\
\hline Case4 & P1,P3 \\
\hline Case5 & P1 \\
\hline Case6 & P1 \\
\hline
\end{tabular}

Table 4: The favorable s-t paths in each case 


\begin{tabular}{|l|r|r|r|r|r|r|}
\hline s-t path & Case1 & Case2 & Case3 & Case4 & Case5 & Case6 \\
\hline P1 & 1 & 1 & 1 & 1 & 1 & 1 \\
\hline P2 & 3 & 3 & 3 & 2 & 3 & 3 \\
\hline P3 & 2 & 2 & 2 & 1 & 2 & 2 \\
\hline P4 & 4 & 5 & 6 & 3 & 5 & 5 \\
\hline P5 & 4 & 4 & 4 & 4 & 6 & 4 \\
\hline P6 & 5 & 6 & 5 & 5 & 7 & 6 \\
\hline P7 & 6 & 7 & 7 & 4 & 4 & 7 \\
\hline
\end{tabular}

Table 5: The ranking scores of $s$-t paths based on $\lambda(P)$

\begin{tabular}{|l|l|r|r|r|r|r|r|}
\hline \multirow{2}{*}{} & Weight restrictions & \multicolumn{5}{|c|}{ CSW } \\
\cline { 3 - 8 } & & $\mathrm{v} 1^{*}$ & $\mathrm{v} 2^{*}$ & \multicolumn{1}{c|}{$3^{*}$} & \multicolumn{1}{c|}{$1^{*}$} & $\mathrm{u}^{*}$ & $\mathrm{u}^{*}$ \\
\hline Case 1 & $\mathrm{v} 2=2 \mathrm{v} 1, \mathrm{u} 1 \geq \mathrm{v} 3, \mathrm{u} 2 \geq 3 \mathrm{u} 1, \mathrm{u} 2 \geq \mathrm{u} 3$ & 0.004823 & 0.009647 & 0.0001 & 0.0001 & 0.565907 & 0.320912 \\
\hline Case 2 & $\mathrm{v} 2 \geq_{\mathrm{v} 1, \mathrm{u} 1 \geq 3 \mathrm{v} 3}$ & 0.199989 & 0.199989 & 0.0001 & 0.0003 & 0.0001 & 5.961904 \\
\hline Case 3 & $\mathrm{v} 1 \geq \mathrm{v} 3, \mathrm{u} 2 \geq \mathrm{v} 3, \mathrm{u} 2 \geq \mathrm{u} 3, \mathrm{u} 3 \leq \mathrm{u} 1$ & 0.002997 & 0.0001 & 0.000109 & 0.0001 & 0.821337 & 0.0001 \\
\hline
\end{tabular}

Table 6: The weight restrictions and generated CSWs

\begin{tabular}{|l|l|l|l|l|l|l|l|l|}
\hline & \multicolumn{1}{|c|}{$\mathrm{P}^{*}$} & $\mathrm{c}_{1}\left(\mathrm{P}^{*}\right)$ & $\mathrm{c}_{2}\left(\mathrm{P}^{*}\right)$ & $\mathrm{c}_{3}\left(\mathrm{P}^{*}\right)$ & $\mathrm{p}_{1}\left(\mathrm{P}^{*}\right)$ & $p_{2}\left(\mathrm{P}^{*}\right)$ & $\mathrm{p}_{3}\left(\mathrm{P}^{*}\right)$ & $\lambda\left(\mathrm{P}^{*}\right)$ \\
\hline Case1 & $P^{*(1)}: 1,5,10,28,30,23$ & 118 & 43.2 & 72330 & 49100 & 3.1 & 2.3 & 0.816466 \\
\hline Case2 & $P^{*(2)}: 1,18,17,23$ & 72 & 26.8 & 48878 & 28000 & 2.2 & 1.4 & 7.899856 \\
\hline Case3 & $P^{*(3)}: 1,5,10,28,30,23$ & 118 & 43.2 & 72330 & 49100 & 3.1 & 2.3 & 0.780849 \\
\hline
\end{tabular}

Table 7: The favorable $s$ - $t$ paths in each case $(s=1, t=23)$

\begin{tabular}{|l|l|l|l|}
\hline $\mathrm{P}^{*}$ & case 1 & case 2 & case 3 \\
\hline$P^{*^{(1)}}=P^{*^{(3)}}$ & 0.816466 & 11.02858 & 0.780849 \\
\hline$P^{*^{(2)}}$ & 0.999329 & 7.899856 & 0.935899 \\
\hline
\end{tabular}

Table 8: Virtual costs for the favorable s-t paths in each case

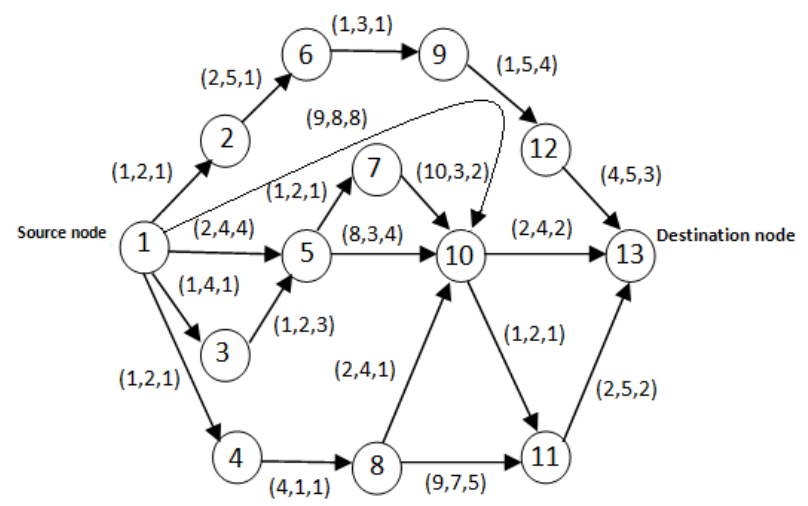

Figure 1: A network with 13 nodes and 19 arcs 


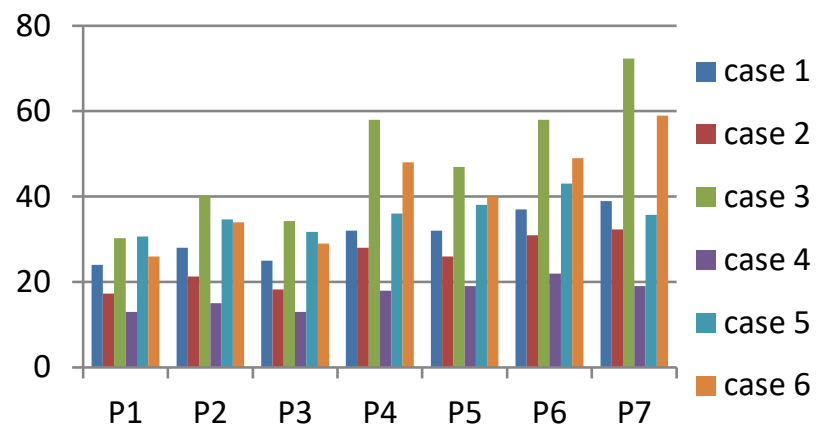

Figure 2: $\lambda(P)$ for each s-t path, $P$, in each case. (in $10^{-4}$ scale)

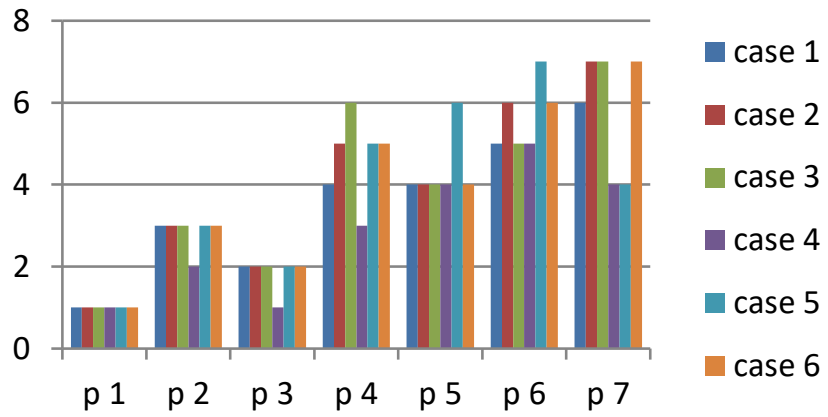

Figure 3: The ranking scores of all $s$-t paths with 6 different cases of weight restrictions

\section{Conclusion}

Heretofore, there are some researches using data envelopment analysis to solve multi-objective shortest path problem. Considering some problems in the previous works, we suggest a new approach using DEA to solve the problem in a more logical way. To this aim, we utilize common weights methodology which is considerable from computational and fairness aspects. Determining a non-dominated path is the main object in our mind. The DEA model serve well to carry through this purpose. Also, DM's preferences, if there are any, are considered to obtain a favorable path among the non-dominated ones from source node to destination node. Furthermore, we can use some polynomial-time shortest path algorithms, like Dijkstra, in this approach.

\section{References}

[1] Ahuja, R. K., Magnanti, T. L. and Orlin, J. B. (1993). Network Flows: Theory, Algorithms, and Applications. New Jersey: Prentice Hall. 
[2] Ahuja, R. K., Mehlhorn, K., Orlin, J. B. and Tarjan, R. E. (1990). Faster algorithms for the shortest path problem. Journal of the Association for Computing Machinery, 37(2), 213-223.

[3] Amirteimoori, A. (2012). An extended shortest path problem: A data envelopment analysis approach. Applied Mathematics Letter, 25, 1839-1843.

[4] Bertsekas, D. P., Guerriero, F. and Musmanno, R. (1996). Parallel asynchronous labelcorrecting methods for shortest paths. Journal of Optimization Theory and Applications, 88(2), 297-320.

[5] Brumbaugh-Smith, J. and Shier, D. (1989). An empirical investigation of some bicriterion shortest path algorithms. European Journal of Operational Research, 43(2), 216-224.

[6] Charnes, A., Cooper, W. W. and Rhodes, E. (1978). Measuring the efficiency of decision making units. European Journal of Operational Research, 2(6), 429-444.

[7] Chen, L. H. and Lu, H. W. (2007). An extended assignment problem considering multiple inputs and outputs. Applied Mathematical Modelling, 31(10), 2239-2248.

[8] Clímaco, J. C., Craveirinha, J. M. and Pascoal, M. M. (2006). An automated reference point-like approach for multicriteria shortest path problems. Journal of Systems Science and Systems Engineering, 15(3), 314-329.

[9] Cook, W. D., Roll, Y. and Kazakov, A. (1990). A DEA Model For Measuring The Relative Eeficiency Of Highway Maintenance Patrols. Information Systems and Operational Research, 28(2), 113-124.

[10] Coutinho-Rodrigues, J. M., Clímaco, J. C. and Current, J. R. (1999). An interactive biobjective shortest path approach: searching for unsupported non-dominated solutions. Computers \& Operations Research, 26 (8), 789-798.

[11] Current, J. R., Revelle, C. S. and Cohon, J. L. (1990). An interactive approach to identify the best compromise solution for two objective shortest path problems. Computers \& Operations Research, 17(2), 187-198.

[12] Dijkstra, E. W. (1959). A note on two problems in connexion with graphs. Numerische mathematik, 1(1), 269-271.

[13] Duque, D., Lozano, L. and Medaglia, A. L. (2015). An exact method for the biobjective shortest path problem for large-scale road networks. European Journal of Operational Research, 242(3), 788-797.

[14] Emrouznejad, A., Zerafat A. L. M. and Ho, W. (2012). An alternative formulation for the fuzzy assignment problem. Journal of the Operational Research Society, 63(1), 59-63.

[15] Guerriero, F. and Musmanno, R. (2001). Label correcting methods to solve multicriteria shortest path problems. Journal of optimization theory and applications, 111(3), 589-613.

[16] Hamacher, H. W., Pedersen, C. R., and Ruzika, S. (2007). Multiple objective minimum cost flow problems: A review. European Journal of Operational Research, 176 (3), 1404-1422.

[17] Hansen, P. (1980). Bicriterion path problems. In Multiple criteria decision making theory and application (pp. 109-127). Berlin: Springer.

[18] Hwang, C. and Masud, A. S. M. (1979). Multiple Objective Decision Making: Methods and Applications. Berlin: Springer-Verlag. 
[19] Liu, F. H. F. and Peng, H. H. (2008). Ranking of units on the DEA frontier with comm on weights. Computers \& Operations Research, 35(5), 1624-1637.

[20] Martins, E. Q. V. (1984). On a multicriteria shortest path problem. European Journal of Operational Research, 16(2), 236-245.

[21] Masoumi, M. M., Lotfi, F. H., and Mobasseri, A. M. (2010). DEA and multi-objective shortest path problems. Contemporary Engineering Sciences, 3(2), 53-62.

[22] Modesti, P. and Sciomachen, A. (1998). A utility measure for finding multi-objective shortest paths in urban multimodal transportation networks1. European Journal of Operational Research, 111(3), 495-508.

[23] Mote, J., Murthy, I. and Olson, D. L. (1991). A parametric approach to solving bi-criterion shortest path problems. European Journal of Operational Research, 53(1), 81-92.

[24] Murthy, I. and Olson, D. L. (1994). An interactive procedure using domination cones for bicriterion shortest path problems. European Journal of Operational Research, 72(2), 417431.

[25] Roll, Y., Cook, W. D. and Golany, B. (1991). Controlling factor weights in data envelopment analysis. IIE transactions, 23(1), 2-9.

[26] Sedeno-Noda, A. and Raith, A. (2015). A Dijkstra-like method computing all extreme supported non-dominated solutions of the biobjective shortest path problem. Computers \& Operations Research, 57, 83-94.

[27] Sinha, A., Korhonen, P., Wallenius, J. and Deb, K. (2014). An interactive evolutionary multi-objective optimization algorithm with a limited number of decision maker calls. European Journal of Operational Research, 233(3), 674-688.

[28] Thompson, R. G., Singleton Jr, F. D., Thrall, R. M. and Smith, B. A. (1986). Comparative site evaluations for locating a high-energy physics lab in Texas. interfaces, 16(6), 35-49.

[29] Zarafat Angiz, M., Saati, S. and Mokhtaran, M (2003). An alternative approach to assignment problem with non-homogeneous costs using common set of weights in DEA. Far East Journal of Applied Mathematics, 10 (1), 29-39. 